\title{
REMANENT MAGNETIZATION OF AMORPHOUS La-Gd-Au ALLOYS WITH HIGH Gd CONTENT
}

S. J. Poon and J. Durand APRIL 1977

A REPORT ON RESEARCH CONDUCTED

UNDER CONTRACT FOR THE

U.S. ENERGY RESEARCH AND DEVELOPMENT ADMINISTRATION

W.W OESTEWED PATENTWISE:

UWLCATION TMEREOF.

W. M. KECK LABORATORY OF

ENGINEERING MATERIALS

CALIFORNIA INSTITUTE OF TECHNOLOGY

PASADENA 


\section{DISCLAIMER}

This report was prepared as an account of work sponsored by an agency of the United States Government. Neither the United States Government nor any agency Thereof, nor any of their employees, makes any warranty, express or implied, or assumes any legal liability or responsibility for the accuracy, completeness, or usefulness of any information, apparatus, product, or process disclosed, or represents that its use would not infringe privately owned rights. Reference herein to any specific commercial product, process, or service by trade name, trademark, manufacturer, or otherwise does not necessarily constitute or imply its endorsement, recommendation, or favoring by the United States Government or any agency thereof. The views and opinions of authors expressed herein do not necessarily state or reflect those of the United States Government or any agency thereof. 


\section{DISCLAIMER}

Portions of this document may be illegible in electronic image products. Images are produced from the best available original document. 
California Institute of Technology

W. M. Keck Laboratory of Engineering Materials

REMANENT MAGNETIZATION OF AMOR PHOUS La-Gd-Au ALLOYS WITH HIGH Gd CONTENT

by

S. J. Poon and J. Durand

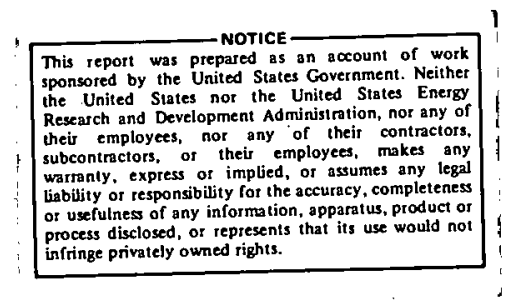

Energy Research and Development Agency Report No.83, under Contract No. $E(04-3)-822$.

Professor Pol Duwez, principal investigator. 


\section{NOTICE}

This report was prepared as an account of work sponsored by the United States Government. Neither the United States nor the Energy Research and Development Administration, nor any of their employees, nor any of their contractors, subcontractors, or their employees, makes any warranty, express or implied, or assumes any legal liability or responsibility for the accuracy, completeness or usefulness of any information, apparatus, product or process disclosed, or represents that its use would not infringe privately-owned rights. 


\begin{abstract}
Results of bulk magnetic measurements are reported for splatcooled amorphous alloys ( $\left.\mathrm{La}_{100-\mathrm{x}} \mathrm{Gd}_{\mathrm{x}}\right)_{80} \mathrm{Au}_{20}$ over the concentrated region between the dilute $(x \leq 1$ at. \%) and the ferromagnetic range ( $x>70$ at. $\%$ ). For $x \leq 40$ at. $\%$ alloys, low field susceptibility maxima occur at temperatures proportional to $\mathbf{x}$, and the reduced remanent magnetization $M_{r s}(T) / x$ depends only on the reduced temperature $T / x$. Our results are analyzed in the spirit of a phenomenological model of uncompensated magnetic clouds. The interplay of the RKKY interaction and of the anisotropic dipolar forces is sufficient to account for the order of magnitude of the remanent magnetization for $\mathrm{x} \leq 40$ alloys.
\end{abstract}


In a preliminary report, we presented the magnetic phase diagram of splat-cooled alloys ( $\left.\mathrm{La}{ }_{100-x} \mathrm{Gd}_{\mathrm{x}}\right)_{80} \mathrm{Au}_{20}$ obtained by complete substitution of $\mathrm{Gd}$ for $\mathrm{La}$ in the amorphous superconductor $\mathrm{La}_{80} \mathrm{Au}_{20^{\circ}}{ }^{1,2}$ The approach to ferromagnetism with increasing Gd content was found to be a rather inhomogeneous and slow process. From zero-field susceptibility measurements and from Arrott-plots at low fields, the $x \geq 80$ at. $\%$ alloys were called "good" ferromagnets. On the other hand, the "dilute" alloys $(0.3 \leq \dot{x} \leq 1)$ were found to behave like canonical spin-glass systems, namely the magnetization and initial susceptibility data follow the universal curves of the scaling prediction. ${ }^{3}$ A detailed analysis of the results for $0.3 \leq x \leq 1$ has been given separately. ${ }^{4}$ The purpose of the present study is to investigate the intermediate region $(1<x \leq 40)$, where the classical scaling laws for $M(H, T)$ are no longer obeyed and to discuss the data in terms of a phenomenological model.

The complex magnetic regimes occurring between the singleimpurity Kondo state and the long-range magnetic order in crystalline alloys of noble metals with $3 d$ impurities ${ }^{5}$ have been the subject of experimental and theoretical interest for at least fifteen years. Recent studies on amorphous concentrated alloys with $\mathrm{Fe}, \mathrm{Co}, \mathrm{Mn}$ impurities 6 showed that the intermediate magnetic regimes are characterized in the amorphous as well as in the crystalline state by the same basic phenomena: sharp susceptibility maxima at low field, is othermal and thermal remanent magnetizations. Relatively fewer systematic studies have been made on both crystalline ${ }^{7}$ and amorphous alloys ${ }^{8}$ containing rare-earth $4 f$-impurities. Some noticeable differences between $3 d$ and $4 f$-alloys are expected. For example, the $4 f$ wave functions are highly 
localized, the RKKY interaction is much weaker, and the crystal field effects might play an important role. On the other hand, in amor phous alloys, both the absence of long-range atomic order and the randomness of local anisotropy are thought to delay the onset of long-range magnetic order. In the case of concentrated La-Gd-Au alloys, our study will be somewhat simplified by the absence of crystal field effects on Gd ions. Some understanding of the magnetic interactions within the wide intermediate magnetic regime may be hopefully gained from a detail investigation of the remanent magnetization. We show that our results for amorphous concentrated alloys, up to $x=40$ at. $\%$, may be analyzed in the light of a phenomenological model recently proposed ${ }^{9}$ for the remanence within the dilute region (typically, 0.1 to 1 at.\%) of crystalline $\mathrm{Au}-\mathrm{Fe}$, Cu-Mn alloys.

Amorphous alloys of nominal Gd concentration $\mathbf{x}=1.25,5,8,12$, $16,20,34,40,50,56,60,70$ and 80 at. $\%$ were obtained by splat cooling technique. ${ }^{2} \mathrm{X}$-ray scanning of the foils indicated patterns (CuK $\left.\alpha\right)$ with broad maxima centered at $\sim 31.5^{\circ}$ with full widths at half maxima of $\sim 4.5^{\circ}$, typical of a glassy metal. From analogy with equivalent amorphous systems ${ }^{10}$, the coordination numbers for Gd are likely to be the same as for La in $\mathrm{La}_{80} \mathrm{Au}_{20}$ (i.e 8 rare earth $+3 \mathrm{Au}$ nearest neighbors). 11 "Zero-field" susceptibility measurements were performed using a standard ac inductance bridge technique. Magnetization was measured between 1.7 and $290^{\circ} \mathrm{K}$ in fields up to $70 \mathrm{kOe}$ using the Faraday method. First, we summarize the common experimental features observed over the whole intermediate concentration range $(1<\mathrm{x} \leq 70)$. Then, for $\mathrm{x} \leq 40$ alloys, where the saturated remanent magnetization $M_{r s} / x$ depends only on $T / x$, we present an analysis of 
our data for $\mathrm{M}_{r s}$ in the spirit of the Tholence-Tournier model. 9

Experimentally, alloys in the intermediate regime $(1<\mathrm{x} \leq 70)$ are characterized by susceptibility maxima $T_{M}$ in low field measurements (see Fig. in ref. 1) and thermomagnetic effects (is othermal and thermal remanent magnetizations). (Fig. 1) The variation of $T_{M}$ as a function of $x$ is linear up to $x=20 \%\left(T_{M}=0.4 \times{ }^{\circ} K\right)$; the departure from linearity is rather small at $\mathrm{x}=40 \%\left(\mathrm{~T}_{\mathrm{M}}=21^{\circ} \mathrm{K}\right)$; for $\mathrm{x}>40 \%, \mathrm{~T}_{\mathrm{M}}$ increases at a faster rate. This is a first indication that the magnetic anisotropy phenomena become more complex in the most concentrated alloys, as it will be confirmed by the remanent magnetization measurements. Relevant parameters for some Gd concentrations are listed in Table 1. Let us note that the values of paramagnetic Curie temperature $\theta_{p}$ obtained from the Curie-Weiss law at high temperature (up to $290^{\circ} \mathrm{K}$ ), which were found to be negative in the dilute region ${ }^{4}(x \leq 1 \%)$, are positive in the concentrated alloys, which characterizes the increasing effects of the ferromagnetic molecular field. The departure between $T_{M}$ and $\theta_{p}$ decreases continuously when the ferromagnetic long-range order is approached: $\left(\theta_{p}-T_{M}\right) / T_{M}$ decreases from 5.8 for $x=5$ at. $\%$, down to $\left(\theta_{p}-T_{c}\right) / T_{c}=0.1$ for $G_{80} A_{20}$. Finally, let us also note that the effective moment per Gd atom as a function of Gd content remains close to the ionic value of $7.94 \mu_{\mathrm{B}}$.

Because of the low values of $T_{M}$ ' we were not able to investigate the thermomagnetic effects for the less concentrated alloys. For the $x>20$ alloys, a systematic study of the remanent magnetization was carried out. In figure 2, two hysteresis loops for the sample $x=40$ obtained by field cooling at $1 \mathrm{kOe}$ and $3 \mathrm{kOe}$ from $\mathrm{T}>\mathrm{T}_{\mathrm{M}}$ to $4.2^{\circ} \mathrm{K}$ are shown. For the $1 \mathrm{kOe}$ field-cooled sample, a displaced loop character- 


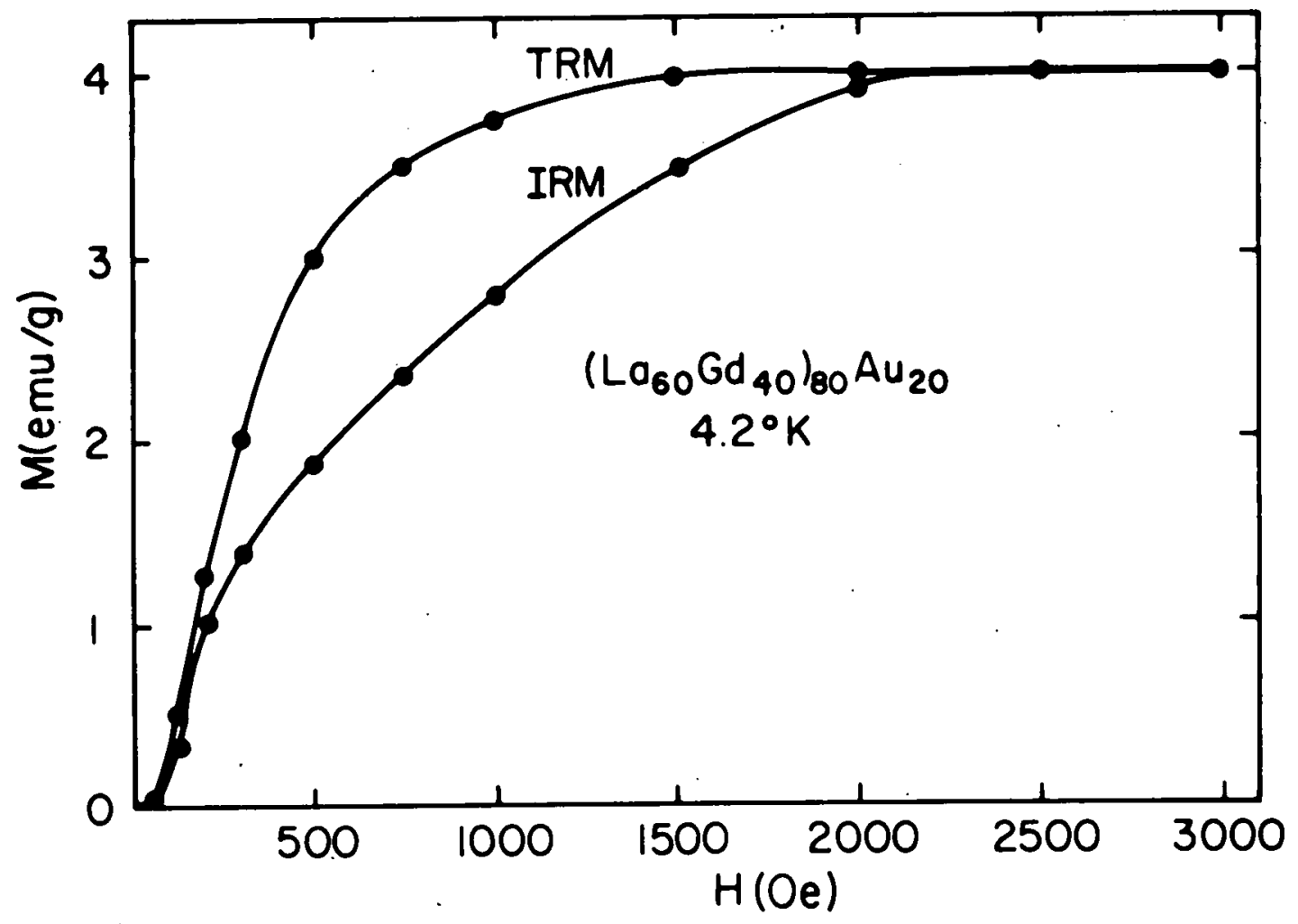

Fig. 1. Is othermal and the rmal remanent magnetizations as a function of cooling field at $4.2^{\circ} \mathrm{K}$ for $\mathrm{x}=40$ alloys. 
Table 1. Magnetic properties of some concentrated amorphous

$$
\left(\text { La } 100-x \mathrm{Gd}_{x}\right)_{80} \mathrm{Au}_{20} \text { alloys. }
$$

$$
\mathrm{T}_{\mathrm{M}}(\mathrm{a}) \quad \theta_{\mathrm{p}} \quad \mathrm{C}_{\mathrm{cW}} \quad \mathrm{M}_{\infty}(0) \quad \mathrm{M}_{\text {eff }}
$$

$x($ at. \% $)\left(\begin{array}{lll}\left.{ }^{\circ} \mathrm{K}\right) & \left({ }^{\circ} \mathrm{K}\right) \quad\left(10^{-3} \mathrm{cgs}\right)\left(\mu_{\mathrm{B}} / \mathrm{Gd} \text { at. }\right) \quad\left(\mu_{\mathrm{B}} / \mathrm{Gd} \text { at. }\right) \\ \hline\end{array}\right.$

$\begin{array}{lcccccc}1.25 & & -1.5 & 0.61 & 8.04 & 8.60 \\ 5 & 1.9 & 13 & 2.18 & 8.14 & 8.12 \\ 20 & 8.5 & 36 & 8.88 & 7.72 & 8.25 \\ 40 & 21 & 72 & 17.62 & 6.57 & 8.30 \\ 80 & 78 & 170 & 30.60 & 7.28 & 7.88 \\ 100 & 150 & 165 & 48.03 & 7.00 & 8.90\end{array}$

(a) The values given for $x=80$ and 100 are the values of $T_{c}$ as determined consistently from Arrott plots and ac bridge measurements. The saturation moment at $0^{\circ} \mathrm{K}, \mathrm{M}_{\infty}(0)$ was determined by a $1 / \mathrm{H}$ extrapolation. The paramagnetic Curie temperature $\theta_{p^{\prime}}$ the Curie-Weiss constants $\mathrm{C}_{\mathrm{CW}}$ and the effective moments $\mathrm{M}_{\text {eff }}$ were obtained from the CurieWeiss law at high temperature (up to $290^{\circ} \mathrm{K}$ ). 


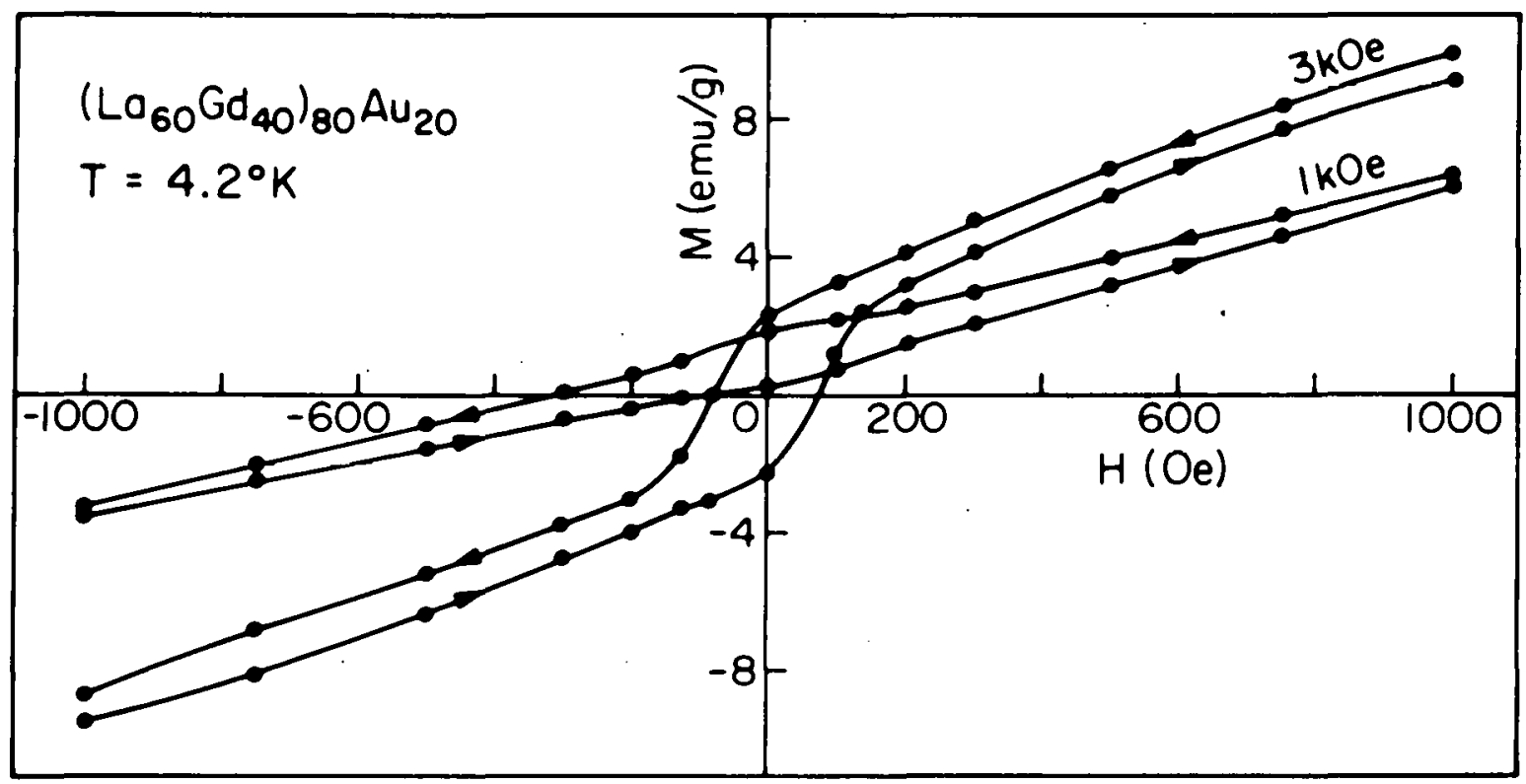

Fig. 2. Magnetization measured at $4.2^{\circ} \mathrm{K}$ vs applied field for $\mathrm{x}=40$ alloys after cooling in fields of 1 and $3 \mathrm{kOe}$. 
istic of the role played by the internal anisotropic field is obtained. When the cooling field is sufficient to saturate the remanent magnetization, as in the $3 \mathrm{kOe}$ case, the loop becomes normal. We studied the temperature dependence of the saturated remanent magnetization. $M_{r s}$ does not vanish at $T_{M}$, but $M_{r s}\left(T_{M}\right) / M_{r s}(0) \simeq 0.5$ for every sample. This fact was already noted for crystalline $\mathrm{V}-\mathrm{Fe}$ alloys. 12 On the other hand, $M_{r s}$ decreases exponentially with increasing temperature. For $x \leq 40$, the variation of $M_{r s} / x$ depends only on $T / x$ and the value of $M_{r s}(0) / M_{\infty}(0)$ remains roughly constant and equal to $\sim 0.05$, suggesting that our data for amorphous concentrated alloys in this range may be analyzed in the light of the Tholence-Tournier-model ${ }^{9}$ for the remanence in dilute $\mathrm{Au}-\mathrm{Fe}$-type alloys. (These regularities are not found in more concentrated alloys).

This phenomenological model was inspired by the analogy between the spin-glass/mictomagnetic phenomena and the magnetic properties (susceptibility maxima, IRM and TRM, relaxation effects) of fine grains in "rock magnetism" as described by Néel. 13 This similarity was pointed out a long time ago. ${ }^{14}$ We summarize the main assumptions and conclusions of this phenomenological description, based on a Ising model. 9 The alloy matrix is assumed to be divided into independent regions to minimize its energy. Each region has an anisotropy energy depending on the number of spins $n$ it contains. The uncompensated moments $\mu n^{\frac{1}{2}}(\mu$ is the moment per spin) of the clouds are assumed to be distributed according to a Gaussian law characterized by its variance $\mu^{2} n_{0^{\circ}}$. The value of $n_{0}$ can be conveniently related to the experimental measurements as $n_{0}=(1 / 2 \pi)\left(M_{\infty}(0) / M_{r s}(0)\right)^{2}$. The applicability of this model relies on the requirement that $M_{\infty}(0) / M_{r s}(0)\left(o r n_{0}\right.$ ) has to 
be rather large and independent of concentration. The anisotropy energy $E_{a}$ of a cloud is as sumed to be proportional to the number $n$ of spins in this cloud: $E_{a}=n_{\mu} \Delta_{a}$, so that in a scaling laws regime, the blocking temperature $T_{B}$ and the width $\Delta_{a}$ of the local anisotropy field of a $n$-cloud will be proportional to $x$. The temperature dependence of $M_{r s}$ may be expressed in this model as

$$
M_{r s}(T)=M_{r s}(0) e^{-\alpha T / x}
$$

where $\alpha$ is correlated to $\Delta_{a}$ as:

$$
\Delta_{\mathrm{a}}=\mathrm{Akx} / \pi \mathrm{n}_{\mathrm{o}} \mu \alpha
$$

A being a coefficient depending on the relaxation time and $k$, the Boltzmainn constant. Finally, the anisotropic part of the dipolar coupling is estimated to be sufficient to give the order of magnitude of $\Delta_{a}$. The different assumptions presented in this model (independent clouds, Gaussian distribution of uncompensated moments, order of magnitude of the $1 / \mathrm{r}^{3}$ anis otropic field distribution) were found to be consistent with the experimental data and other features of $\mathrm{Au}-\mathrm{Fe}$ and Cu-Mn alloys in the dilute region: the relaxation effects on $M_{r s}$ $\left(M_{r s}(t) \alpha \log t\right)$, the fact that below the saturation at a given temperature TRM is larger than IRM, the exponential decrease of $M_{r s}(T) / x$ as a function of $T / x$. The different model parameters are determined quantitatively, so far as their orders of magnitude are concerned. In addition, the model predicts that the irreversible properties might be significantly enhanced in systems with weak RKKY interaction, as Gd 
diluted in normal metals. We would like to check this model prediction.

In Fig. 3, is shown the variation of $M_{r s}(T) / x$ as a function of $T / x$ for alloys with $x=20$ and 40 at. $\%$ Gd. So equation (1) is well verified, and the value of $\alpha$ is found to be 1.4 at. $\% \mathrm{Gd} /{ }^{\circ} \mathrm{K} . \mathrm{M}_{\mathrm{rs}}(0)$ can also be obtained from Fig. 3 to give the ratio $M_{r s}(0) / M_{\infty}(0) \approx 5.6 \cdot 10^{-2}$. This value is larger than that obtained in dilute Au-Fe alloys ${ }^{9}\left(2 \cdot 10^{-2}\right)$. In comparison, the value equals 0.2 in a concentrated $\mathrm{Cu}_{81} \mathrm{Mn}_{19}$ alloy. 15 Consequently, the value we obtain for $n_{0}$ is rather small $n_{0} \simeq 50$ ( $n_{0}$ equals 350 to 500 in dilute AuFe alloys). 9 The experimental value of $T_{M}$ can be interpreted as the blocking temperature $T_{B}$ for the mean clouds. The number $n$ of spins of these clouds may be determined by two different ways. First, from the Gaussian distribution of the moments, one derives $n=n_{0} \pi \alpha T_{M} / x$ which gives $n=135$ spins from our experimental values of $\mathrm{n}_{0}, \alpha$, and $\mathrm{T}_{\mathrm{M}}$. On the other hand, if the anisotropic dipolar coupling is sufficient to justify the presence of potential barriers, $\mathrm{n}$ is also given approximately by the ratio of the amplitudes of the RKKY and dipolar couplings ${ }^{9}, \mathrm{n}=0.85 \mathrm{~V}{ }_{\mathrm{o}} / \mu_{\mathrm{B}}^{2}$, where $V_{0}$ is the strength of the RKKY interaction $V(r)=V_{0}\left(\cos 2 k_{F} r\right) / r^{3}$. Using $V_{0}=0.2 \times 10^{-37} \mathrm{erg} \mathrm{cm}^{3}$ determined ${ }^{4}$ from the approach to saturation of magnetization for $x \leq 1$, we obtain $n=210$ spins. The qualitative agreement between the two values of $n$ obtained from model predictions is satisfactory up to $x=40$. The low values of $n$ as compared with the dilute Au-Fe, CuMn systems $\left(n=10^{4}\right)$ originate from the fact that the RKKY exchange interaction $(s-f)$ is much weaker than in the s-d alloys. Large relaxation effects a re also observed in our alloys, as can be seen by comparing Fig. 1 and Fig. 2: $M(H=0)$ of Fig. 2 is reduced to half of the saturated TRM of Fig. 1. The time of 


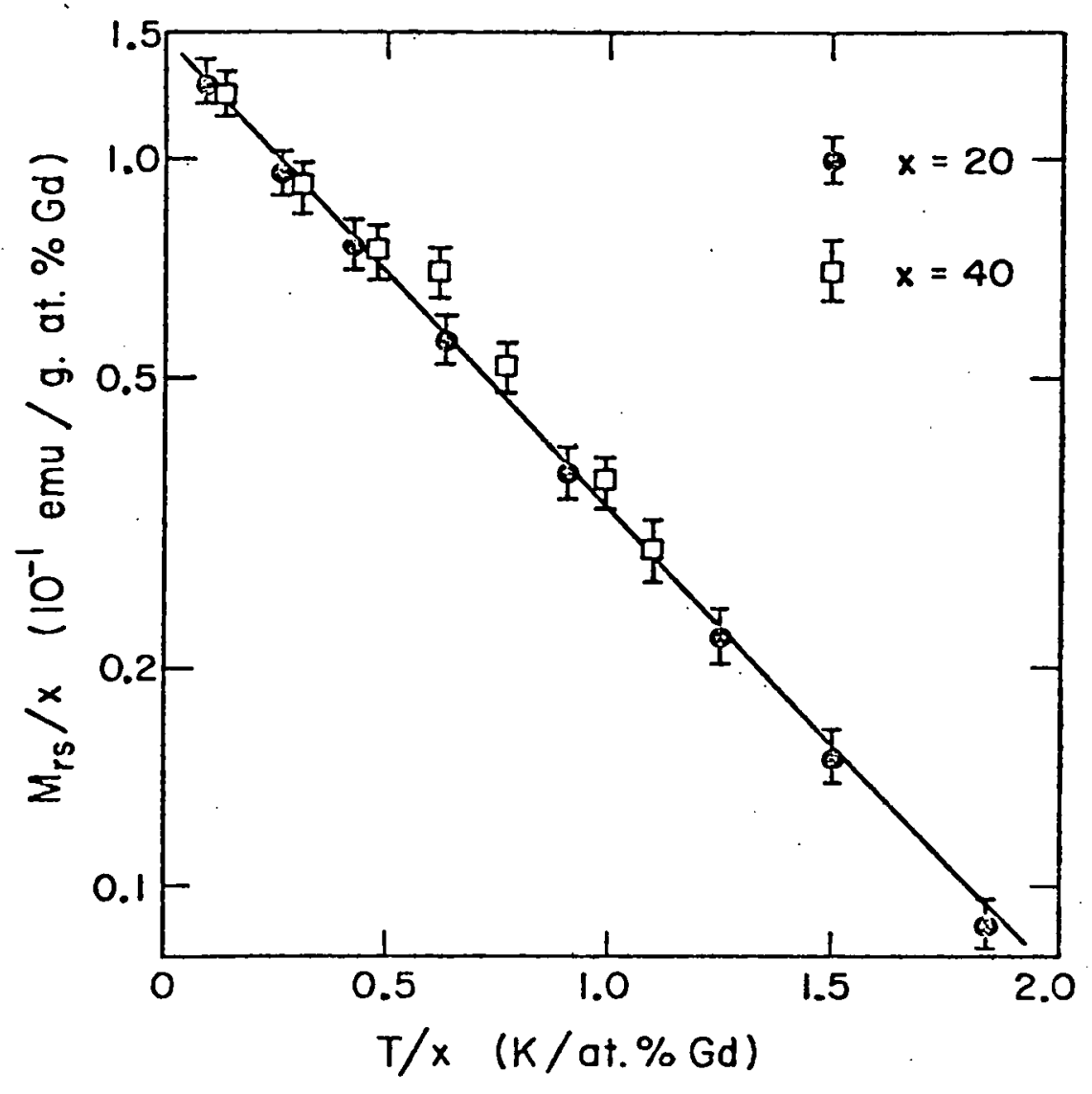

Fig. 3. Reduced saturated remanent magnetization (log scale) vs reduced temperature for the $x=20$ and 40 alloys. 
measurements of the former quantity is $\sim 2000 \mathrm{sec}$, while it is $\sim 200$ sec for the latter. Similar relaxation effects are also observed in IRM at $4.2^{\circ} \mathrm{K}$. When such time scale of the measurements is applied to the case of $\underline{\mathrm{Au}-\mathrm{Fe} \text { alloys }}{ }^{9}$, it only accounts for $10 \%$ reduction in the TRM at $4.2^{\circ} \mathrm{K}$. Thus, for the present system which favors small clouds, the relaxation time is considerably smaller than in $3 \mathrm{~d}$ canonical alloys. Taking into account this short relaxation time, the order of magnitude of the width $\Delta_{a}$ of the distribution of the local anisotropy fields on each impurity can be estimated to be 200 Oe for $x=40$ (see equation 2). The order of magnitude of $\Delta_{a}$ is the same as for the Au-Fe alloys.

In summary, the model predictions concerning the irreversible properties of Gd in normal metals is found to be partially fulfilled in our amorphous alloys of La-Au with high Gd content. The value we obtained for $\mathrm{M}_{\mathrm{rs}}(0) / \mathrm{M}_{\infty}(0)$ is larger than for dilute $\mathrm{Cu}-\mathrm{Mn}$ alloys, but it is smaller than for concentrated $\mathrm{Cu}_{81} \mathrm{Mn}_{19}$ alloys. The most remarkable feature in our study is that the concentration range for the spin-glass/mictomagnetic phenomena in amorphous La-Gd-Au is enhanced appreciably as compared with crystalline 3 d-alloys. The region where the scaling laws for $M(H, T)$ are valid is about the same as for Au-Fe alloys $\left(x \leq 1\right.$ at. $\%$ ). But $T_{M}$ is proportional to $x$ and $M_{r s}(T) / x$ is a unique function of $T / x$ up to $x=40$. It has been already observed in crystalline Mo-Fe alloys ${ }^{16}$ that the scaling laws for the specific heat a re still holding over a concentration range where the scaling laws for $\mathrm{M}(\mathrm{H}, \mathrm{T})$ are not valid anymore. The observed departure may be explained in our case as follows. The scaling law for $M(H, T)$ relies strictly on the indirect interaction between two well separated spins; while, according to our analysis, $M_{r s}(T)$ is correlated 
to a cloud of $\mathrm{n}$ spins, $\mathrm{n}$ being relatively large (130 to 200 ) and independent of concentration. 17 The dependence of $M_{r s}(T) / x$ on $T / x$ leads to the following conclusions. First, the existence of a scaling law for $M_{r s}(T)$ rules out the hypothesis that the remanence would be due to some chemical cluster effects in these concentrated amorphous alloys. Second, the agreement between the two $n$ values implies the existence of a $1 / r^{3}$ interaction creating the potential barriers which retain a large number of moments in the direction of the field previously applied. Of course, for alloys with such high concentrations of Gd, it is impossible to deny the existence of other possible anisotropic forces. However, for our alloys, the $1 / \mathrm{r}^{3}$ anisotropic dipolar interactions seems to be sufficient to account for the remanence up to $x=40$ at. $\% \mathrm{Gd}$. Above $\mathrm{x}=40$, the interactions between clouds are more complex in nature. Finally, let us note that the concentration we estimated to be the critical concentration for long-range ferromagnetic order $(x=80)$ is far above the critical concentration predicted by the percolation theories. ${ }^{18}$ Counting only the R.E. nearest neighbors as the relevant coordination number ${ }^{11}$ (i.e. $Z=8$ ), the percolation limit lies between 20 to 24 at. $\%$ Gd, according to different models. ${ }^{18}$ However, the present case of RKKY interactions must be distinguished from transition metal alloys where the $d-d$ overlaps are important for the occurrence of ferromagnetism. It must be added that in amor phous alloys, the fluctuations in bond lengths are significant and even the couplings among nearest-neighboring $\mathrm{Gd}$ atoms might be statistical in nature. Thus the delay in the onset of ferromagnetism in amorphous La-Gd-Au alloys might be favored by both the strong localization of $4 \mathrm{f}$ electrons leading to oscillatory RKKY interaction and the amorphous structure 
of these alloys. But, from comparison with crystalline cases ${ }^{7}$, the former effect is believed to be predominant.

ACKNOWLEDGMENT - The authors wish to thank Professor Pol Duwez for his interest and support throughout this work. Gratitude is also extended to Dr. R. Tournier for discussions and for making available his preprints to the authors. 


\section{REFERENCES}

1. Poon, S. J. and Durand, J., Second International Symposium on Amorphous Magnetism, Troy, New York 1976 (to be published).

2. Johnson, W. L., Poon, S. J. and Duwez, P., Phys. Rev. B 11 , $150(1975)$.

3. Souletie, J. and Tournier, R., J. Low Temp. Phys. 1,95 (1969).

4. Poon, S. J. and Durand, J., Sol. State Commun., 21, 793 (1977); id. 21,999 (1977).

5. See the reviews of Beck, P. A., Met. Trans. 2, 2015 (1971); Tournier, R., in L. T. '13, Boulder, Colorado, 1972, Timmerhaus et al. (Eds.) (Plenum Press, New York, 1974) vol. 2, p. 257;

Coles, B. R., in Amorphous Magnetism, Hooper and de Graaf (Eds.) (Plenum Press, New York, 1973), p. 169; Perrier, J. P., and Tholence, J. L., J. Phys. (Paris) 35, C4, 163 (1974); Mydosh, J.A., AIP Conf. Proc. 24, 131 (1974) and in ref. 1.

6. Sharon, T. E., and Tsuei, C. C., Phys. Rev. B 5, 1047 (1972) (PdP-Fe); Zentko, A., Duhaj, P., Potochki, L., Tima, T., and Bansky, J., Phys. Stat. Solidi (a) 31, K4l (1975) (PdSi-Fe, PdSi-Co); Sherwood, R. C., Gyorgy, E. M., Leamy, H. J., and Chen, H. S., AIP Conf. Proc. 34, 325 (1976) (PBA 1-FeMn); Amamou, A., in ref. 1 (NiPB-Mn); Durand, J., in ref. 1 (NiPB-Fe).

7. Sarkissian, B. V. B., and Coles, B. R., Commun. Phys. 1,17 (1976); Bennett, M. H., and Coles, B. R., Physica 86-88B, 844 (1977). 
8. Mizoguchi, T., McGuire, T. R., Kirkpatrick, S., and Gambino, R. J., Physica 86-88R, 783 (1977).

9. Tholence, J. L., and Tournier, R., J. Phys. (Paris) 35, C4, 229 (1974), and Physica 86-88B, 877 (1977); Holtzberg, F., Tholence, J. L., and Tournier, R., in ref. 1 .

10. Cargill, G. S. III, in Seitz et al. (Eds.), Solid State Phys. 30, (Academic Press, New York, 1975) p. 225.

11. Logan, J., Scripta Metallurgica 9, 379 (1975).

12. Claus, H., Phys. Rev. Lett. 34, 26 (1975).

13. Néel, L., Ann. Geoph. $\underline{5}, 99$ (1954); Adv. Phys. $\underline{4}, 191$ (1955).

14. Lutes, O. S., and Schmit, J. L., Phys. Rev. 125, 433 (1962); Tournier, R., and Weil, L., J. Phys. Rad. 23, 522 (1962).

15. Claus, H., and Kouvel, J. S., Sol. State Commun. 17, 1553 (1975).

16. Amamou, A., Caudron, R., Costa, P., Friedt, J. M., Gautier, F., and Loegel, B., J. Phys. F 6,2371 (1976).

17. In Au-Fe alloys, $M_{r s}(T) / x$ does not scale with $T / x$ for $x>1$ at. $\%$. The temperature and concentration dependence of $M_{r s}$ over both the dilute and the concentrated regions can be accounted for by a phenomenological model which does not make any assumption about the origin of the blocking energy (see Tholence, J. L., thesis, G renoble, 1973). 
18. Elliott, R. J., J. Phys. Chem. Solids 16, 165 (1960); Sykes, M. F., and Essam, J. W., Phys. Rev. 133A, 310 (1964); Sata, H., and Kikuchi, R., AIP Conf. Proc. 18, 605 (1974). 